\title{
SNR Improvement of QEPAS System by Preamplifier Circuit Optimization and Frequency Locked Technique
}

\author{
Qinduan $\mathrm{ZHANG}^{1}$, Jun $\mathrm{CHANG}^{1}{ }^{*}$, Zongliang $\mathrm{WANG}^{2}$, Fupeng $\mathrm{WANG}^{1}$, \\ Fengting JIANG ${ }^{1}$, and Mengyao WANG ${ }^{1}$ \\ ${ }^{1}$ School of Information Science and Engineering and Shandong Provincial Key Laboratory of Laser Technology and \\ Application, Shandong University, Jinan, 250100, China \\ ${ }^{2}$ School of Physics Science and Information Technology and Shandong Key Laboratory of Optical Communication \\ Science and Technology, Liaocheng University, Liaocheng, 252059, China \\ *Corresponding author: Jun CHANG_E-mail: changjun@sdu.edu.cn
}

\begin{abstract}
Preamplifier circuit noise is of great importance in quartz enhanced photoacoustic spectroscopy (QEPAS) system. In this paper, several noise sources are evaluated and discussed in detail. Based on the noise characteristics, the corresponding noise reduction method is proposed. In addition, a frequency locked technique is introduced to further optimize the QEPAS system noise and improve signal, which achieves a better performance than the conventional frequency scan method. As a result, the signal-to-noise ratio (SNR) could be increased 14 times by utilizing frequency locked technique and numerical averaging technique in the QEPAS system for water vapor detection.
\end{abstract}

Keywords: QEPAS system; preamplifier circuit; frequency locked method; noise optimization

Citation: Qinduan ZHANG, Jun CHANG, Zongliang WANG, Fupeng WANG, Fengting JIANG, and Mengyao WANG, "SNR Improvement of QEPAS System by Preamplifier Circuit Optimization and Frequency Locked Technique," Photonic Sensors, 2018, 8(2): 127-133.

\section{Introduction}

The quartz enhanced photoacoustic spectroscopy (QEPAS) technique is presented as a common method to detect gas concentration such as $\mathrm{CO}, \mathrm{NO}_{2}$, $\mathrm{N}_{2} \mathrm{O}, \mathrm{SO}_{2}$, and $\mathrm{CH}_{4}$ in the last dozen years. It has many advantages compared with other conventional gas detection technologies such as small size, low cost, strong anti-interference, and high sensitivity. So there is an increasing research boom in QEPAS gas sensing field, in which the second harmonic method is the main detection means. A superposition of low frequency scanning ramp and high frequency modulation signal is usually used to drive the laser.
The frequency of high frequency modulation signal is half of the resonance frequency of quartz tuning fork (QTF). The modulated optical signal produces gas vibration which contains second harmonic signal by infrared spectrum absorption. Then second harmonic acoustical signal is transformed into current signal by QTF. As a result, the current signal generated by the QTF is converted to a voltage signal by the preamplifier circuit. A lock-in amplifier is used to extract the second harmonic waveform [1-5]. The preamplifier circuit is an important section of QEPAS system because it directly determines the quality of signal [6]. Usually, the more complex the circuit is, the more noise will

Received: 19 September 2017 / Revised: 11 March 2018

(C) The Author(s) 2018. This article is published with open access at Springerlink.com

DOI: $10.1007 / \mathrm{s} 13320-018-0468-y$

Article type: Regular 
be added. However, few people have deeply researched it.

In this paper, operational amplifier (OPA) CA3140E (Intersil, USA) is utilized as an example to conduct a comprehensive analysis of the preamplifier circuit noise and measure the noise contribution of the various parts of the circuit. Based on the analysis, series of corresponding noise reduction methods are proposed to optimize the preamplifier circuit.

A frequency locked method based on the wavelength modulation technology and signal detection theory is also presented. Different from low frequency sawtooth signal that scans over absorption peak as shown in Fig. 1(a), the central frequency of laser is locked at the absorption peak $1368.597 \mathrm{~nm}$ in our QEPAS system as shown in Fig. 1(b). Therefore, noise can be reduced by compressing the filter bandwidth of the lock-in amplifier. And by means of numerical averaging technology, noise will be further reduced.

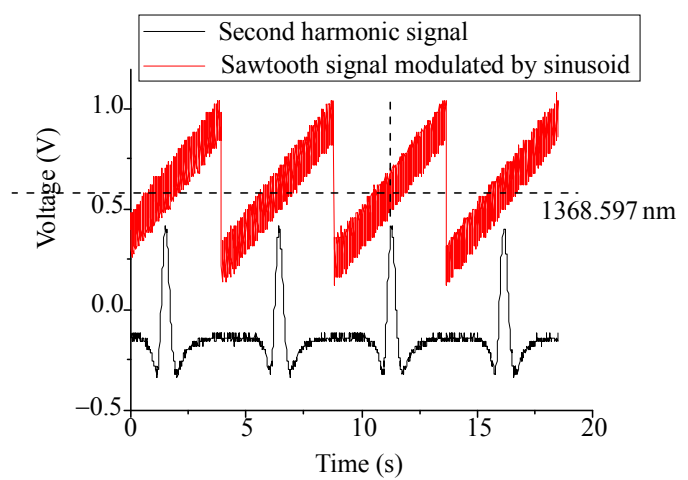

(a)

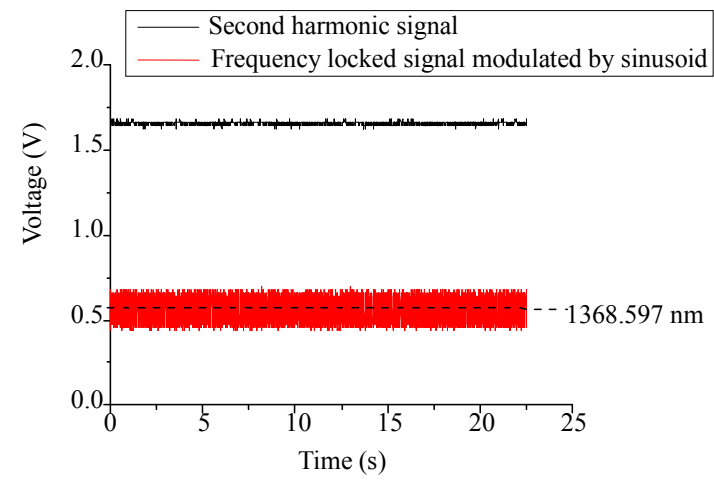

(b)

Fig. 1 Laser driving voltage and the corresponding second harmonic signal: (a) frequency scan method and (b) frequency locked method.
Experimental results show that the signal-to-noise ratio (SNR) increases no less than 14 times of the frequency scan method on condition that the real-time constraint is satisfied.

\section{Theory analysis}

\subsection{Noise analysis of preamplifier circuit}

In this section, how to utilize the noise model to calculate the total output noise will be discussed. In Fig. 2(a), the input noise includes OPA voltage noise $V_{2}$, OPA current noise $I$, resistance noise $V_{1}$ produced by $R_{1}$, and $V_{3}$ produced by $R_{f}$. The noise model in Fig. 2(a) can be converted to Fig. 2(b).

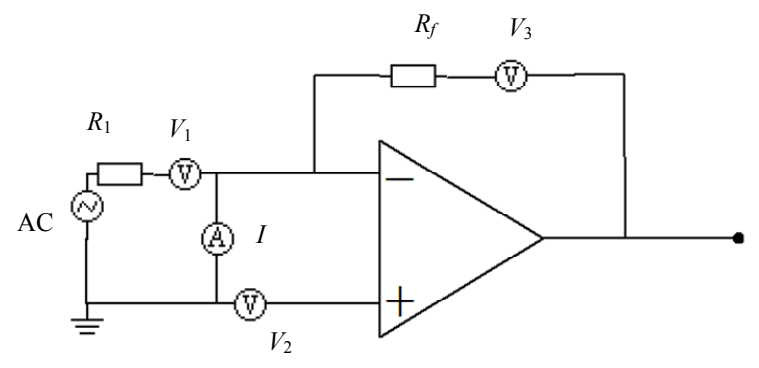

(a)

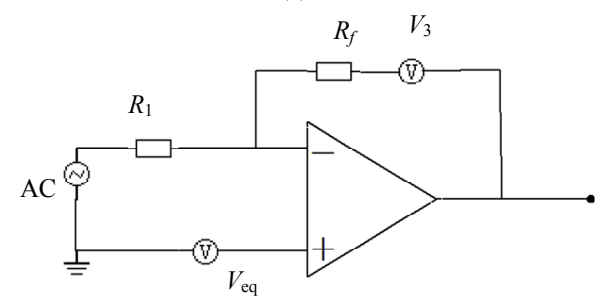

(b)

Fig. 2 Noise model: (a) inverting amplifying circuit and (b) equivalent circuit.

As long as the input reference noises are provided, the output noise voltage could be calculated through the noise gain (NG). The NG equation is easily obtained:

$$
\mathrm{NG}=\frac{R_{f}}{R_{1}}+1 .
$$

The output voltage generated by $V_{2}$ can be directly obtained:

$$
V_{2_{-} \text {output }}=V_{2} \cdot \mathrm{NG}=V_{2} \cdot\left(\frac{R_{f}}{R_{1}}+1\right) \text {. }
$$

The input voltage noise generated by $I$ is

$$
V_{I}=I \cdot R_{\text {eq }}
$$


where $R_{\uparrow}=\frac{R_{1} \cdot R_{f}}{R_{1}+R_{f}}$.

The output voltage generated by $I$ can be got by

$$
V_{I_{-} \text {output }}=V_{I} \cdot \text { Noise_gain }=I \cdot R_{f} \text {. }
$$

At last we calculate the thermal noise of the resistors $R_{1}$ and $R_{f}$. The resistor noise mainly includes three parts: Johnson noise, contact noise, and shot noise. In general, the metal film resistor noise can be approximately expressed in Johnson noise as

$$
V_{R_{\mathrm{eq}}}=V_{1}+V_{3}=\sqrt{4 k T R_{\mathrm{eq}} \cdot \Delta f}
$$

where $k$ is the Boltzmann constant, $T$ is thermodynamic temperature, and $\Delta f$ is the bandwidth.

$$
V_{R_{\text {eq }- \text { output }}}=V_{R_{\text {eq }}} \cdot \mathrm{NG}=\sqrt{4 k T \frac{R_{f}\left(R_{1}+R_{f}\right)}{R_{1}} \Delta f} .
$$

For the preamplifier circuit, $R_{f}$ is far more larger than $R_{1}$,

$$
V_{R_{\text {eq }} \text { output }} \approx \frac{R_{f}}{\sqrt{R_{1}}} \cdot \sqrt{4 k_{B} T \Delta f} .
$$

So the total equal output noise is the addition of $V_{2 \_ \text {output }}, V_{\text {I_output, }}$ and $V_{\text {eq_output }}[7]$ :

$$
V_{\text {eq_output }}=\sqrt{V_{2_{-} \text {output }}^{2}+V_{I_{\text {_output }}}^{2}+V_{\mathrm{R}_{\text {eq_ }} \text { output }}^{2}} .
$$

\subsection{QTF resonant theory}

The equivalent circuit of QTF can be shown in Fig. 3. The equivalent impedance of the QTF $R$ represents the loss of mechanical vibration, the equivalent inductance of the QTF $L$ represents vibration quality, the equivalent capacitance of the QTF $C$ represents the mechanical elastic, and $C_{0}$ represents the stray capacitance and parasitic capacitance caused by the surface metal wire of the QTF $[8,9]$. It is usually considered that resonant current of QTF reaches a maximum at series resonant frequency $\omega_{0}$, therefore according to the solution of second-order RCL series resonant circuit, the time constant can be expressed as: $\alpha=-R / 2 L$.

The energy accumulation time $t$ (the resonant energy is 0.707 times of the maximum resonant energy) can be shown as (9):

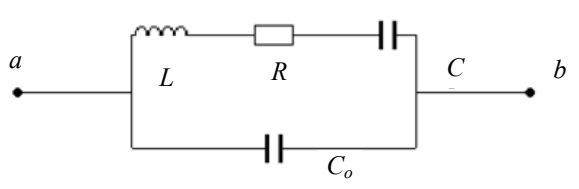

Fig. 3 Equivalent circuit of QTF.

$$
e^{\alpha t}=e^{-1} \text {. }
$$

According to the equation of the time constant $\alpha$ and (9), the energy accumulation time $t$ can be shown as follows:

$$
t=\frac{2 L}{R} .
$$

Besides, the quality factor $Q$ can be shown as follows:

$$
Q=\frac{\rho}{R}=\frac{\omega_{0} L}{R} .
$$

The energy accumulation time $t$ can be solved by $Q$ and $f_{0}$ based on (10) and (11)

$$
t=\frac{Q}{\pi f_{0}}
$$

where $f_{0}=\omega_{0} / 2 \pi$.

Most often, $Q$ is about 8000 in the atmosphere pressure $[10,11]$. According to (12), the energy accumulation time $t$ is about $0.077 \mathrm{~s}$. This is only a value on condition that resonant energy is attached to 0.707 times of the maximum resonant energy. By inference, the conservative energy accumulation time would be several hundreds of milliseconds.

Thus when DFB-LD is driven by a low frequency sawtooth signal, QTF cannot reach the maximum of resonance owing to that the laser is scanned over the absorption line of the measured gas too quickly. On the other hand, continuous variation of wavelength leads to signal spectrum broadening and energy dispersion. So if energy loss of the second harmonic signal is not desirable, the bandwidth of low-pass filter of lock-in amplifier cannot be set too narrow.

\section{Experimental verification}

\subsection{Noise measurement of preamplifier circuit}

To analyze the contribution of each part of noise source in preamplifier circuit, several experiments were conducted by utilizing 7230 General Purpose 
DSP Lock-in Amplifier (AMETEK USA). In order to measure the noise in the vicinity of QTF central frequency, central frequency $32768 \mathrm{~Hz}$ and bandwidth $0.8 \mathrm{~Hz}$ were set to measure the output noises. Experimental circuit is shown in Fig. 2(b), and $\mathrm{AC}$ source was used instead of wire.

First of all, we only changed $R_{1}$ and measured the output $V_{n}$. $V_{n}$ was equal to $2.5 \mu \mathrm{V}$ while $R_{1}$ was disconnected. This noise value could be thought to be a contribution of $R_{f}$ and OPA.

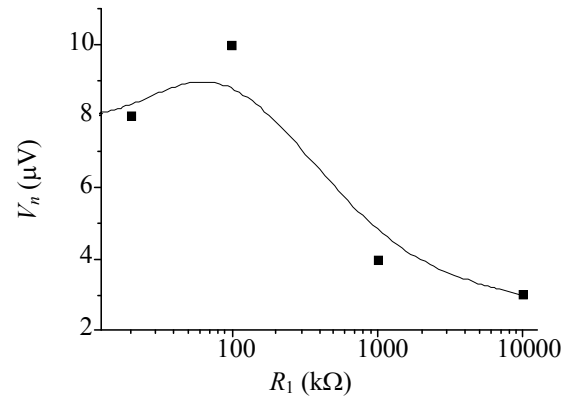

Fig. 4 Output noise $V_{n}$ ranges with $R_{1}$ on condition that $R_{f}=$ $10 \mathrm{M} \Omega$.

Output $V_{n}$ generated by $R_{1}$ can be shown as follows:

$$
V_{R_{1 \_} \text {output }}=\frac{R_{f}}{R_{1}} \cdot V_{R_{1}} .
$$

Based on (5), the following equation is yielded:

$$
V_{R_{1}-\text { output }}=\frac{R_{f}}{\sqrt{R_{1}}} \cdot \sqrt{4 k_{B} T \Delta f} .
$$

Considering that the gain-bandwidth product (GBP) of OPA CA3140E is about $4.5 \mathrm{MHz}$, the maximum magnification can be calculated to be 137 times, so as shown in Fig. 4 , on condition that $R_{1}$ is small, $V_{\mathrm{R} 1}$ output increases with the rising of $R_{1}$ and will not become larger all the time because the noise magnification starts to decrease linearly and the noise of $R_{1}$ increases only by $\sqrt{R_{1}}$. This conclusion may hint whether the preamplifier circuit is in a low noise state.

Then the simulation experiment of QTF internal resistor noise amplifying was carried out. In order to simulate QTF internal resistor, an experiment measuring QTF internal resistor should be completed. The experimental circuit of QTF internal resistor is shown in Fig. 5. A $5 \mathrm{mV}$ sine signal was input in its resonant frequency, and the output was
$500 \mathrm{mV}$. So according to the noise gain relation and theory of series resonance, it could be considered that the QTF internal equivalent resistor was $R_{1}=$ $1 / 100 \cdot R_{f}=100 \mathrm{k} \Omega$.

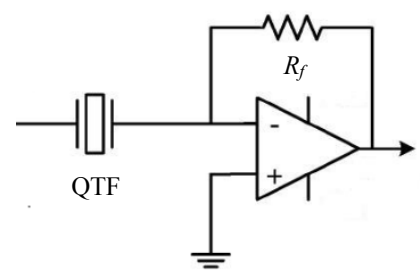

Fig. 5 Measurement circuit of QTF internal resistor.

So $R_{1}=100 \mathrm{k} \Omega$ was chosen as QTF, and we only changed $R_{f}$. The output noise was measured as shown in Fig. 6. It could be seen that the output noise becomes larger nearly linear with the $R_{f}$ increasing. So the $R_{f}$ less than $30 \mathrm{M} \Omega$ could be suitable value in QTF preamplifier circuit using OPA CA3140E which not only reaches high gain but also prevents signal distortion because of limited GBP.

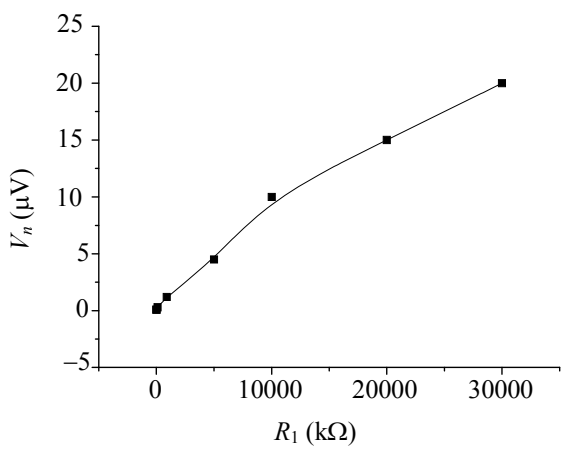

Fig. 6 Simulation experiment of QTF internal resistor noise amplifying.

While the noise of preamplifier circuit with QTF mentioned in Section 3.2 is about 2 times of the preamplifier circuit with $R_{1}$ instead of QTF as shown in Fig. 6. Thus it could be concluded that not only noise contribution of QTF consisted in the internal equivalent resistor but some other reasons such as air turbulence.

According to the above description, the output noise $V_{n}$ consisted in QTF, $R_{f}$, and OPA. According to (4), no matter QTF consisted the output noise of OPA, the current noise $I$ could be shown as $V_{\text {I_output }}=$ $I \cdot R_{f}$. So the noise contribution of $R_{f}$ and OPA was about $2.5 \mu \mathrm{V}$. As a result, it could be found that the noise contribution of QTF was much more than that 
of $R_{f}$ and OPA.

For OPA, input-referred current noise and input-referred voltage noise also play an important part in keeping high SNR in the QTF preamplifier circuit. It is necessary to choose the OPA of low input-referred current noise and input-referred voltage noise to reduce the output noise. GBP should also be considered which determines the maximum magnification of the second harmonic signal. In order to obtain high magnification, the OPA should have high GBP. High precision $R_{f}$ with low noise and temperature drift would also increase SNR partly.

\subsection{Comparative experiment of QTF resonance intensity}

Our experimental system applied for water vapor concentration detection aiming at verifying the superiority of frequency locked method is shown as Fig. 7. The wavelength of absorption peak was chosen at $1368.597 \mathrm{~nm}$, and the modulation frequency was set into $16384 \mathrm{~Hz}(1 / 2$ of the QTF resonant frequency). A function generator (FY2300A, Feel Tech, China) was used to generate the modulation signal. DC/scan signal to drive laser was produced by ARM LPC1758 (NXP Semiconductor, Netherlands), and 7230 Lock-in Amplifier was utilized in our experimental system to recover second harmonic signal.

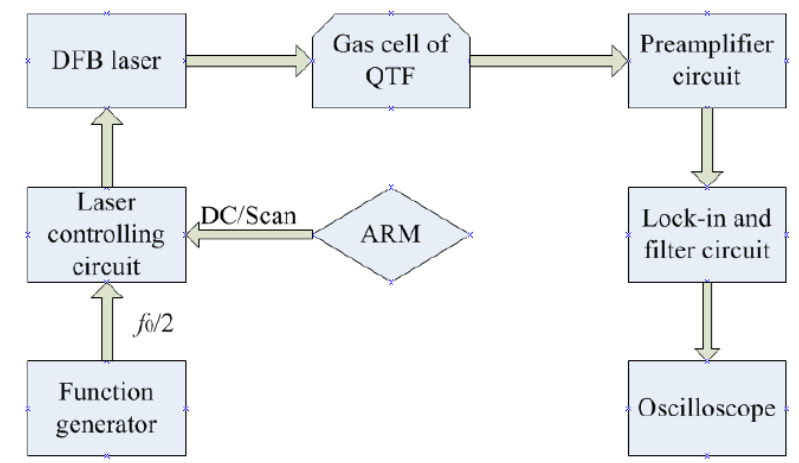

Fig. 7 Schematic of experimental verification system.

In order to verify the difference of QTF resonance intensity between the frequency scan method and frequency locked method, the second harmonic signal was measured in different scan periods by 7230 Lock-in Amplifier whose bandwidth of low-pass filter was set to $32 \mathrm{~Hz}$. The result of measurement is shown as Fig. 8. Bandwidth $32 \mathrm{~Hz}$ was set to prevent the effect of signal spectrum broadening and energy dispersion caused by frequency scan on comparative experiment of QTF resonance intensity according to Section 2.2.

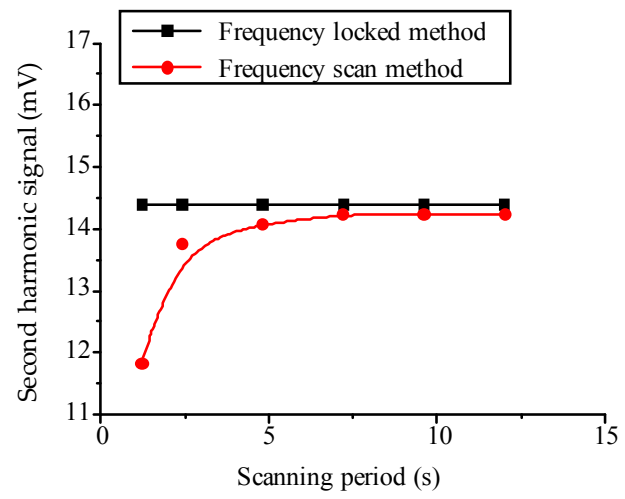

Fig. 8 Second harmonic signal of frequency locked method and frequency scan method.

From Fig. 8, it could be concluded that the QTF resonance intensity of frequency locked method is in a high level, and only when the scan period is more than $4.8 \mathrm{~s}$ can the QTF resonance intensity of frequency scan method achieve maximum.

\subsection{Comparative experiment of signal intensity and noise measurement in different bandwidth}

Since the second harmonic signal of the frequency scan method was proportional to the filter bandwidth of the lock-in amplifier, the filter bandwidth of the lock-in amplifier could not be set too narrow. Otherwise, the second harmonic signal would be lost, if the filter bandwidth was too wide, the noise would be too large. Figure 9 depicts the second harmonic signal of frequency locked method and frequency scan method in different bandwidths. In order to eliminate the effect of QTF resonance intensity demonstrated by Fig. 8 , the scan period of $4.8 \mathrm{~s}$ was an ideal value for this experiment.

The experimental results showed that the signal amplitude of the frequency locked method had no relation with the filter bandwidth of the lock-in amplifier. But the frequency scan method achieved maximum only when the filter bandwidth was greater than $1.5 \mathrm{~Hz}$. 


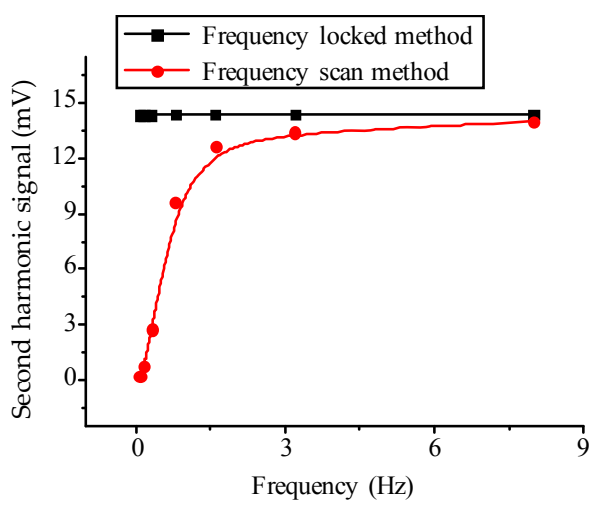

Fig. 9 Second harmonic signal of frequency locked method and frequency scan method in different bandwidths.

The filter bandwidth of lock-in amplifier not only affected the signal intensity, but also affected the noise. According to thermal noise (5), thermal noise would decrease with the $\Delta f$ narrowing. An experiment was carried out to verify this formula, and the result is shown in Fig. 10.

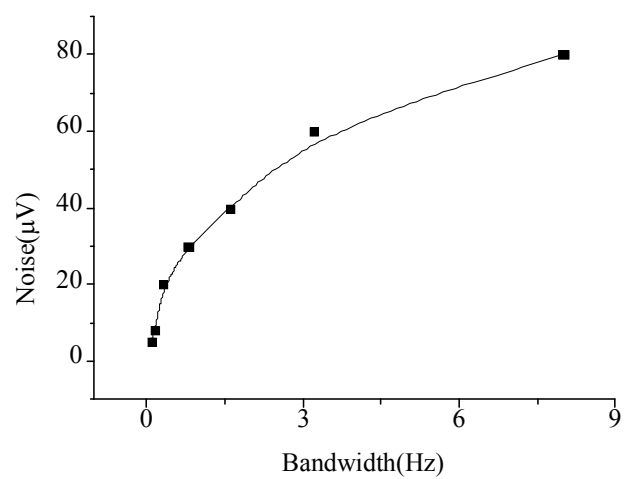

Fig. 10 Noise measurement in different low-pass filter bandwidths.

\subsection{QEPAS system of vapor concentration detection based on frequency locked method and numerical averaging technique}

The QEPAS system of vapor concentration detection we designed is shown in Fig. 11. Different from experiment above, chip AD630 (ANALOG DEVICES, USA) and peripheral circuit [12] we designed independently were utilized as lock-in and filter circuit instead of 7230 General Purpose DSP Lock-in Amplifier. It is nothing less than 7230 but its costs are far lower. The bandwidth of low-pass filter was set to $1 \mathrm{~Hz}$. The output of lock-in and filter circuit was acquired into ARM. Acquired data was processed by a $\mathrm{C} \#$ program which was downloaded into ARM and then transferred to computer.

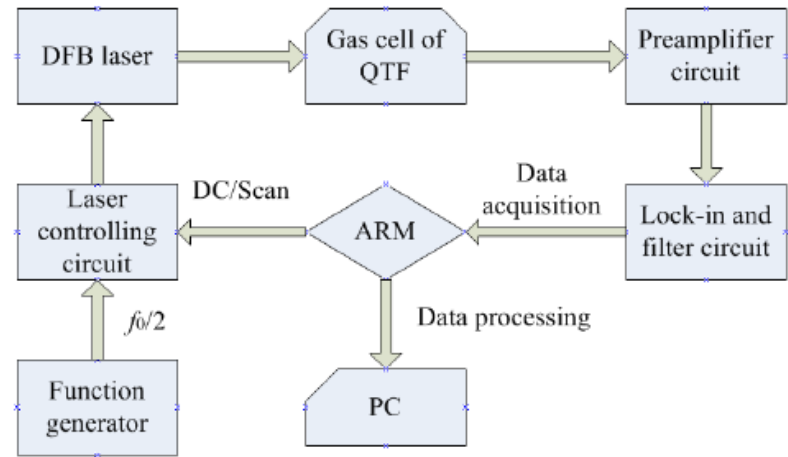

Fig. 11 Schematic of QEPAS system of vapor concentration detection based on frequency locked method and numerical averaging technique.

The second harmonic signal was detected and processed by frequency locked method and frequency scan method, respectively. To satisfy the real-time constraints, the scan period of frequency scan method was set to $1.2 \mathrm{~s}$. So there would be only one point acquired per $1.2 \mathrm{~s}$ by frequency scan method while in this $1.2 \mathrm{~s}$ time 6000 points could be collected and averaged by ARM by the frequency locked method. Numerical averaging partly optimized away the data fluctuation caused by noise.

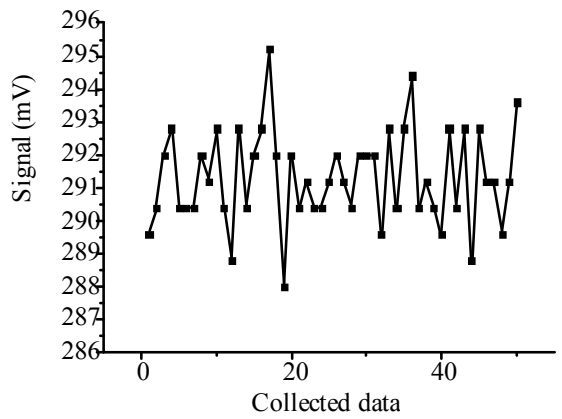

(a)

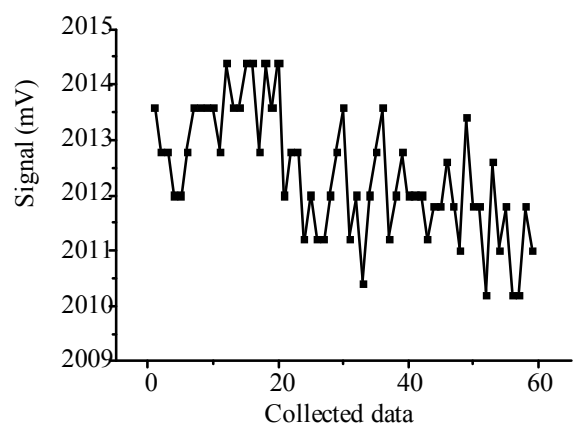

(b)

Fig. 12 Data acquisition: (a) frequency scan method and (b) frequency locked method.

The collected data in PC are shown in Fig. 12. 
The signal of the frequency locked method is about 7 times larger than that of the frequency scan method, and data fluctuation of frequency locked method is about half of the frequency scan method. As a result, the SNR could be increased 14 times by frequency locked and numerical averaging technique.

\section{Conclusions}

In this paper, the preamplifier circuit noise is measured and analyzed by utilizing CA3140, which shows the main noise source to be QTF and may also instruct us on how to choose $R_{f}$ and OPA. Comparative experiment between the frequency locked method and frequency scan method demonstrates that the QTF resonance intensity is always in the maximum state and spectrum broadening and output noise will reach to the minimum by the frequency locked method. On the condition of quick response, our QEPAS system of water vapor concentration detection based on frequency locked method and numerical averaging technique achieves about 500 SNR, which is 14 times of frequency scan method.

\section{Acknowledgment}

This work was supported by the National Natural Science Foundation of China (Grant Nos. $61405105 \&$ 61475085), the Shandong Provincial Natural Science Foundation, China (Grant No. ZR2014FQ015), Science and Technology Development Project of Shandong Province (Grant No. 2014GGX101007), and the Fundamental Research Funds of Shandong University (Grant Nos. 2014HW016 \& 2014YQ011).

Open Access This article is distributed under the terms of the Creative Commons Attribution 4.0 International License (http://creativecommons.org/ licenses/by/4.0/), which permits unrestricted use, distribution, and reproduction in any medium, provided you give appropriate credit to the original author(s) and the source, provide a link to the Creative Commons license, and indicate if changes were made.

\section{References}

[1] W. Schippers, E. Gershnabel, J. Burgmeier, O. Katz, U. Willer, I. S. Averbukh, et al., "Stimulated Raman rotational photoacoustic spectroscopy using a quartz tuning fork and femtosecond excitation," Applied Physics B, 2011, 105(2): 203-211.

[2] Y. N. Liu, J. Chang, J. Lian, Z. J. Liu, Q. Wang, and C. G. Zhu, "A time difference method for measurement of phase shift between distributed feedback laser diode (DFB-LD) output wavelength and intensity," Sensors, 2015, 15(7): 16153-16161.

[3] Y. N. Liu, J. Chang, J. Lian, Z. J. Liu, Q. Wang, and Z. G. Qin, "Quartz-enhanced photoacoustic spectroscopy with right-angle prism," Sensors, 2016, 16(2): 1-7.

[4] A. A. Kosterev, F. K. Tittel, D. V. Serebryakov, A. L. Malinovsky, and I. V. Morozov, "Applications of quartz tuning fork in spectroscopic gas sensing," Review of Scientific Instruments, 2005, 76(4): 043105-1-043105-9.

[5] Q. D. Zhang, J. Chang, F. P. Wang, Z. L. Wang, Y. L. Xie, and W. H. Gong, "Improvement in QEPAS system utilizing a second harmonic based wavelength calibration technique," Optics Communications, 2018, 415: 25-30.

[6] K. Matsui, OPA application skills 100 cases. Beijing, China: Science Press, 2006: 65-75.

[7] A. Kay, "Analysis and measurement of intrinsic noise in op amp circuits," Global Electronics China, 2010, 6: 41-44.

[8] P. Patimisco, G. Scamarcio, F. K. Tittel, and V. Spagnolo, "Quartz-enhanced photoacoustic spectroscopy: a review," Sensors, 2014, 14(4): 6165-6206.

[9] R. D. Grober, J. Acimovic, J. Schuck, D. Hessman, P. J. Kindlemann, J. Hespanha, et al., "Fundamental limits to force detection using quartz tuning forks," Review of Scientific Instruments, 2000, 71(7): 2776-2780.

[10] A. A. Kosterev, Y. A. Bakhirkin, and F. K. Tittel, "Ultrasensitive gas detection by quartz-enhanced photoacoustic spectroscopy in the fundamental, molecular absorption bands region," Applied Physics $B, 2005,80(1):$ 133-138.

[11] A. A. Kosterev, Y. A. Bakhirkin, R. F. Curl, and F. K. Tittel, "Quartz-enhanced photoacoustic spectroscopy," Optics Letters, 2002, 27(21): 1902-1904.

[12] X. Chen, J. Chang, F. P. Wang, Z. L. Wang, W. Wei, Y. Y. Liu, et al., "A portable analog lock-in amplifier for accurate phase measurement and application in high-precision optical oxygen concentration detection," Photonic Sensors, 2017, 7(1): 27-36. 